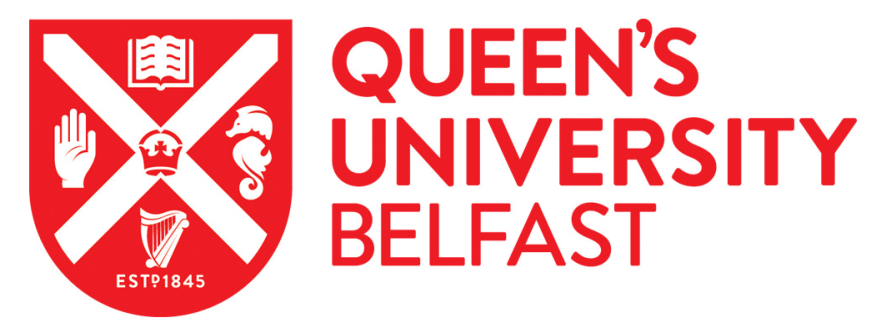

\title{
Fading Characterization of UE to Ceiling-Mounted Access Point Communications at $60 \mathrm{GHz}$
}

Yoo, S. K., Cotton, S. L., Chun, Y. J., \& Scanlon, W. G. (2018). Fading Characterization of UE to CeilingMounted Access Point Communications at $60 \mathrm{GHz}$. In 12th European Conference on Antennas and Propagation (EuCAP) 2018: Proceedings The Institution of Engineering and Technology. https://doi.org/10.1049/cp.2018.0483

Published in:

12th European Conference on Antennas and Propagation (EuCAP) 2018: Proceedings

Document Version:

Publisher's PDF, also known as Version of record

Queen's University Belfast - Research Portal:

Link to publication record in Queen's University Belfast Research Portal

Publisher rights

(c) 2018 IEEE.

This work is made available online in accordance with the publisher's policies. Please refer to any applicable terms of use of the publisher.

\section{General rights}

Copyright for the publications made accessible via the Queen's University Belfast Research Portal is retained by the author(s) and / or other copyright owners and it is a condition of accessing these publications that users recognise and abide by the legal requirements associated with these rights.

Take down policy

The Research Portal is Queen's institutional repository that provides access to Queen's research output. Every effort has been made to ensure that content in the Research Portal does not infringe any person's rights, or applicable UK laws. If you discover content in the Research Portal that you believe breaches copyright or violates any law, please contact openaccess@qub.ac.uk. 


\title{
Fading Characterization of UE to Ceiling-Mounted Access Point Communications at $60 \mathrm{GHz}$
}

\author{
Seong Ki Yoo, Simon L. Cotton, Young Jin Chun and William G. Scanlon \\ Centre for Wireless Innovation, ECIT Institute \\ Queen's University Belfast, BT3 9DT, UK \\ \{sk.yoo, simon.cotton, y.chun, w.scanlon\}@qub.ac.uk
}

\begin{abstract}
This paper presents an investigation of the first- and second-order statistics of the small-scale fading observed in short range indoor millimeter-wave channels between a user equipment (UE) and a ceiling-mounted access point (AP) operating at $60 \mathrm{GHz}$. In this study, three UE positions associated with common use cases were considered: making a voice call (head), sending a text message or operating an app (hand) and carrying a device (pocket). For the mobile line of sight (LOS) and nonLOS (NLOS) channel measurements, the user walked towards and away from the AP within an indoor hallway environment. The first- and second-order statistics of the Rice and Nakagami$m$ fading models were employed to characterize the smallscale fading behavior experienced in the UE-to-AP channels. Overall, they were shown to provide a good agreement with the measured channel data. Using the Akaike information criterion, the Nakagami- $m$ distribution was favored for the majority of the cases considered in this study.
\end{abstract}

Index Terms-average fade duration, fading characterization, indoor mm-wave channel; level crossing rate; small-scale fading.

\section{INTRODUCTION}

The use of millimeter-wave (mm-wave) operating frequencies is one of approaches currently being employed to satisfy the demand for higher data rates in future wireless networks [1]. Within the mm-wave region of the spectrum, the unlicensed $60 \mathrm{GHz}$ band has been proposed as a promising candidate for multi-gigabit short range indoor wireless communications [2]. Nonetheless, like all wireless systems, indoor mmwave communications can be impacted by multipath fading and shadowing (or equivalently blockage). This is especially important when mm-wave devices are operated near the human body, while the user is mobile. One simple strategy targeted at reducing the magnitude of these effects is to mount access points (APs), or equivalently evolved NodeBs (eNBs) in the case of cellular systems, at ceiling level. This setup aims to improve the probability of achieving a line of sight (LOS) between the user equipment (UE) and the AP. In the process, reducing the potential impact of blockages and multipath fading caused by obstacles in the local environment including humans. It is worth highlighting that the multipath fading is mainly considered in this study.

A number of studies have been performed to investigate the statistical characteristics of the small-scale fading experienced in indoor mm-wave communications channels at $60 \mathrm{GHz}[3-$ 5]. For example, the small-scale fading observed in off-body communications channels was characterized in [3] using the
Rice and Nakagami- $m$ distributions, while in [4] a similar methodology was used to study UE-to-eNB channels. In [5], it was found that the Rice fading model provided the best fit to the measurement data collected in LOS conditions within an indoor laboratory environment.

While these studies have provided important insights into the statistical characteristics of the small-scale fading experienced in mm-wave communications channels at $60 \mathrm{GHz}$, they have been limited to analysis of first-order statistics including the probability density function (PDF) and cumulative distribution function (CDF). Unlike the first-order statistics, second-order statistics such as the level crossing rate (LCR) and average fade duration (AFD) give a direct indication of the rate of change of the fading with respect to time. Consequently, they are essential for the development of many of the important attributes of wireless systems including the design and selection of error correcting techniques, the optimization of interleaver depth, system throughput analysis and channel modeling [6]. To address these issues, in this paper, we perform a fading characterization of some indoor mm-wave channels which exist between a UE and a ceiling mounted AP operating at $60 \mathrm{GHz}$. In particular we focus on characterizing the second-order statistics of these channels using the Rice and Nakagami- $m$ fading models. Since the UE is operated in close proximity to the user's body the user effects are included in mm-wave UE-to-AP channels considered in this paper.

\section{MEASUREMEnT SyStem AND EXPERIMENTS}

\section{A. Measurement System}

The hypothetical mm-wave UE used for the measurements consisted of a Hittite HMC6000LP711E transmitter (TX) module $^{1}$, which features a $+7.5 \mathrm{dBi}$ antenna in-package. The system was configured to transmit a continuous wave signal centered at $60.05 \mathrm{GHz}$ using at an Equivalent Isotropically Radiated Power (EIRP) of $+10.9 \mathrm{dBm}$. To emulate a hypothetical UE, the TX module was securely fixed to the inside of a compact acrylonitrile butadiene styrene enclosure $(80 \mathrm{~mm}$ $\times 80 \mathrm{~mm} \times 20 \mathrm{~mm}$ ) as shown in Fig. 1 .

The hypothetical mm-wave AP used for the measurements consisted of a Hittite HMC6001LP711E receiver (RX) mod-

\footnotetext{
${ }^{1}$ http://www.analog.com/media/en/technical-documentation/datasheets/hmc6000.pdf (visited on 10/21/2017).
} 


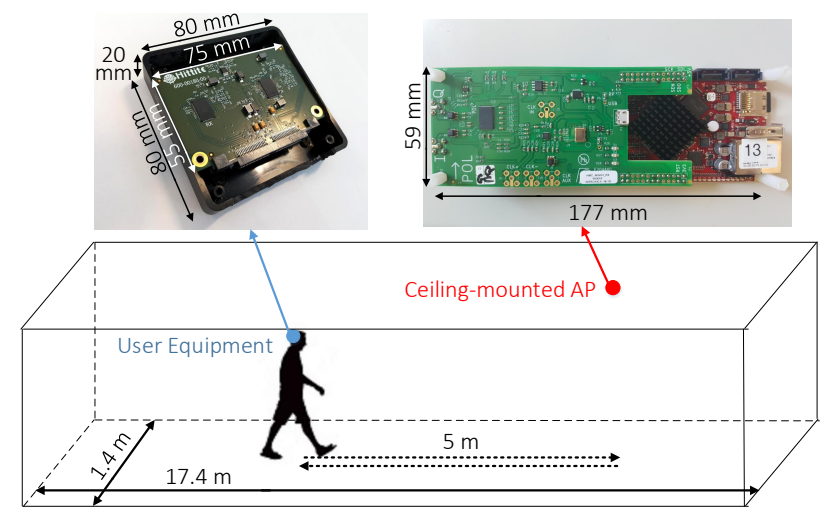

Fig. 1: Hypothetical UE and AP used for the measurements in an indoor hallway environment.

ule $^{2}$ (Fig. 1). This unit also features a $+7.5 \mathrm{dBi}$ antenna in-package with normalized free-space radiation patterns as depicted in Fig. 2. The $50 \mathrm{MHz}$ intermediate frequency (IF) signal outputted by the RX module was recorded using a v1.4 Red Pitaya data acquisition platform ${ }^{3}$. The Red Pitaya v1.4 contains a 14-bit, $125 \mathrm{Msps}$ analog-to-digital converter (ADC) which is connected to a field programmable gate array (FPGA). The FPGA unit was programmed with a custom direct digital down conversion implementation based on the embedded SDR receiver described in [7]. This implementation provided an effective channel sampling frequency of $2 \mathrm{kHz}$ and a receive bandwidth of $86 \mathrm{kHz}$. The AP was placed above the ceiling tile with the antenna boresight oriented towards the floor.

\section{B. Measurement Environment and Use Cases}

The measurements were performed in an indoor hallway environment as shown in Fig. 1, which is located on the $1^{\text {st }}$ floor of the ECIT institute, Queen's University, Belfast, UK. The indoor environment featured metal studded dry walls with a metal tiled floor covered with polypropylene-fiber, rubber backed carpet tiles, and metal ceiling with mineral fiber tiles and recessed louvered luminaries suspended $2.70 \mathrm{~m}$ above floor level. The hallway environment was unoccupied for the duration of the measurements.

During the measurements, the UE was held at different body locations by an adult male of height of $1.83 \mathrm{~m}$ and mass $78 \mathrm{~kg}$ according to three UE positions which are likely to be representative of everyday UE usage. These were: (1) the right-head region to imitate making a voice call; (2) two hands in front of his body to imitate sending a text message or operating an app; (3) the right-front pocket of his clothing to imitate carrying a device. Consequently, the antenna boresight was oriented towards the wall, ceiling (or the user's face) and AP for the head, hand and pocket positions, respectively. In this study, we considered two different channel conditions,

\footnotetext{
${ }^{2}$ http://www.analog.com/media/en/technical-documentation/datasheets/hmc6001.pdf (visited on 10/21/2017).

${ }^{3}$ This is manufactured by Red Pitaya [https://redpitaya.com/ (visited on 10/21/2017)].
}

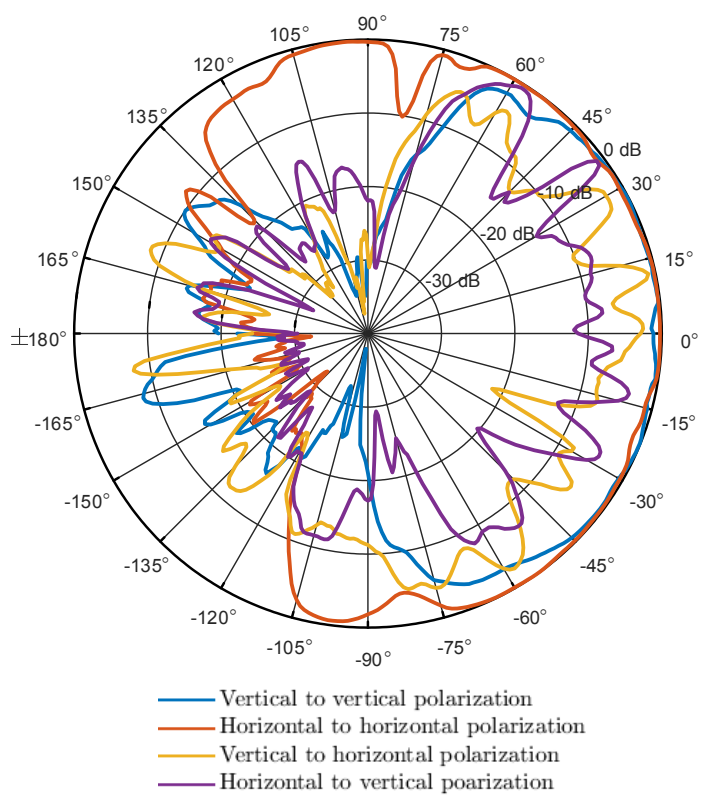

Fig. 2: Normalized free-space radiation patterns with respect to the maximum gain observed in the case of horizontal to horizontal polarization. ${ }^{4}$

namely: (1) mobile LOS and (2) mobile NLOS where the user walked towards and away from the hypothetical AP in a straight line respectively. To improve the validity and robustness of the parameter estimates obtained in this study, all the measurements were repeated five times. The walking distance was $5 \mathrm{~m}$ and the average walking speed maintained by the user throughout all of the experiments was found to be approximately $1 \mathrm{~m} / \mathrm{s}$.

\section{FIRST- AND SECOND-ORDER STATISTICS}

The Rice fading model has traditionally been used to describe small-scale fading for LOS channel conditions, while the Nakagami- $m$ fading model is synonymous with the characterization of NLOS channels. Both the Rice and Nakagami-m fading models have previously been found to provide a good fit to measurement data obtained in $60 \mathrm{GHz}$ communication channels [3], [4].

\section{A. First-order Statistics: $P D F$ and $C D F$}

The PDF and CDF of the received signal envelope, $R$, over a Rice fading channel can be expressed as follows [9]

$$
f_{R}(r)=\frac{2(K+1) r I_{0}\left(2 \sqrt{\frac{K(K+1) r^{2}}{\Omega}}\right)}{\Omega \exp \left(K+\frac{(K+1) r^{2}}{\Omega}\right)}
$$

and

${ }^{4}$ The radiation pattern measurements were performed in the anechoic chamber described in [8]. A separation distance of $1.2 \mathrm{~m}$ was maintained between the TX and RX. The TX was positioned on a height adjustable wooden tripod while the RX was placed on the turn table with the turn table rotating $360^{\circ}$ in a clockwise direction with each step of $1^{\circ}$. 


$$
F_{R}(r)=1-Q\left(\sqrt{2 K}, \sqrt{\frac{2(K+1) r^{2}}{\Omega}}\right)
$$

where $I_{0}(\cdot)$ represents the modified Bessel function of the first kind with order zero and $Q(\cdot)$ denotes the Marcum $Q$ function. In terms of its physical interpretation, the $K$ factor $(K>0)$ is defined as the ratio between the power in the dominant component and the power in the scattered component whereas $\Omega=\mathbb{E}\left[r^{2}\right]$ is the mean signal power with $\mathbb{E}[\cdot]$ denoting the expectation operator.

In a Nakagami- $m$ fading channel, the PDF and CDF of the signal envelope, $R$, can be expressed as [10]

$$
f_{R}(r)=\frac{2 m^{m} r^{2 m-1}}{\Gamma(m) \Omega^{m}} \exp \left(-\frac{m r^{2}}{\Omega}\right)
$$

and

$$
F_{R}(r)=\frac{\gamma\left(m, m r^{2} / \Omega\right)}{\Gamma(m)}
$$

where $\Gamma(\cdot)$ and $\gamma(\cdot, \cdot)$ denote the gamma and lower incomplete gamma functions, respectively. In terms of its physical interpretation, $m$ represents the fading severity parameter and $\Omega$ denotes the mean signal power.

\section{B. Second-order Statistics: LCR and AFD}

The LCR is defined as the average number of times a fading envelope crosses a given signal level with a positive slope within a certain period of time. Similarly, AFD is defined as the average time duration that a fading envelope is found below a given signal level. The LCR and AFD of the received signal envelope, $R$, over a Rice fading channel can be expressed as [9]

$$
N_{R}(r)=\frac{f_{m} \sqrt{2 \pi(K+1) r^{2}} I_{0}\left(2 \sqrt{\frac{K(K+1) r^{2}}{\Omega}}\right)}{\sqrt{\Omega} \exp \left(K+\frac{(K+1) r^{2}}{\Omega}\right)}
$$

and

$$
T_{R}(r)=\frac{\exp \left(K+\frac{(1+K) r^{2}}{\Omega}\right)\left[1-Q\left(\sqrt{2 K}, \sqrt{2 \frac{(1+K) r^{2}}{\Omega}}\right)\right]}{f_{m} \sqrt{\frac{2 \pi(K+1) r^{2}}{\Omega}} I_{0}\left(2 \sqrt{\frac{K(1+K) r^{2}}{\Omega}}\right)}
$$

where $f_{m}$ is the maximum Doppler shift given in $\mathrm{Hz}$. The corresponding LCR and AFD of the received signal envelope, $R$, over a Nakagami- $m$ fading channel can be expressed as [10]

$$
N_{R}(r)=\frac{f_{m} \sqrt{2 \pi}}{\Gamma(m)}\left(\frac{m r^{2}}{\Omega}\right)^{m-\frac{1}{2}} \exp \left(-\frac{m r^{2}}{\Omega}\right)
$$

and

$$
T_{R}(r)=\frac{\gamma\left(m, m r^{2} / \Omega\right)}{f_{m} \sqrt{2 \pi}}\left(\frac{\Omega}{m r^{2}}\right)^{2 m-1} \exp \left(\frac{m r^{2}}{\Omega}\right) .
$$

\section{Results}

To extract the small-scale fading from the channel data for analysis, all the measurements were normalized with respect to the local mean. The local mean level was calculated by averaging over a distance of 10 wavelengths (or equivalently 100 samples). The parameter estimates for the Rice ${ }^{5}$ and Nakagami- $m$ fading models were obtained using maximum likelihood estimation (MLE) available in MATLAB. Table I shows the mean value of the parameter estimates for the Rice and Nakagami- $m$ models for all of the considered cases averaged over the five repeated trials.

It is clear that there existed a strong dominant signal component $(K>1)$ for the LOS channel conditions over all of the UE positions. When comparing UE positions, the pocket position had higher $K$ parameter estimates than those for the head and hand positions, suggesting that there existed stronger dominant signal components in the pocket-to-AP channels compared to others. This observation is supported by the parameters $s$ and $\sigma$ shown in Table I. More specifically, the dominant signal power $\left(s^{2}\right)$ for the pocket position was greater than those for head and hand positions while the scattered signal power $\left(2 \sigma^{2}\right)$ for the pocket position was smaller compared to those for other positions. Interestingly, a strong dominant signal component existed even for the NLOS hand case, suggesting that the hand UE position typically had a partial optical (or quasi) LOS with the AP. Similarly, when comparing the $m$ parameters for all of the UE positions, the highest $m$ parameter was observed at the pocket and hand positions for the LOS and NLOS channel conditions respectively. Nevertheless, across all the LOS and NLOS scenarios, the $m$ parameters were greater than 1 , indicating that the NLOS channel conditions were subject to less severe fading than those experienced in Rayleigh fading channels.

As an example of the model fitting process, Fig. 3 provides the Rice and Nakagami- $m$ CDFs for the LOS scenarios during the $2^{\text {nd }}$ trial and NLOS scenarios during the $1^{\text {st }}$ trial along with the respective empirical CDFs. It can be easily seen that the Rice and Nakagami- $m$ fading models were shown to provide a good agreement with the measurement data. To ascertain the most probable model between Rice and Nakagami- $m$ distributions, the second-order Akaike information criterion $\left(A I C_{c}\right)$ was used in a similar manner to the analysis employed in [11]. According to the $A I C_{c}$ results shown in Table I, it was found that the Nakagami- $m$ distribution was chosen as the best model with the exception of the LOS pocket case. For the LOS scenarios, the direct signal path between the UE and AP was obscured by the user's hand for the head and hand positions whereas there existed the direct signal link between UE and AP for the pocket position. Because of this, the Nakagami-m distribution was favored for the LOS head and hand cases. For the NLOS scenarios, the UE-to-AP channels for all of the UE

\footnotetext{
${ }^{5}$ The non-centrality $(s)$ and scaling $(\sigma)$ parameters were estimated along with the PDF given in $[9$, Eq. (2.53)] and then the parameters $K$ and $\Omega$ were obtained from the parameters $s$ and $\sigma$ using the relationship given in $[9, \mathrm{Eq}$ (2.57)].
} 
TABLE I: Average parameter estimates for the Rice and Nakagami- $m$ fading models alongside the estimated maximum Doppler shift $\left(f_{m}\right)$ and $A I C_{c}$ rank

\begin{tabular}{cccccccccccc}
\hline \multirow{2}{*}{ Channel Conditions } & \multirow{2}{*}{ UE Positions } & \multicolumn{1}{c}{ Rice } & \multicolumn{4}{c}{ Nakagami- $m$} \\
& & $s$ & $\sigma$ & $K$ & $\Omega$ & $f_{m}(\mathrm{~Hz})$ & Rank & $m$ & $\Omega$ & $f_{m}(\mathrm{~Hz})$ & Rank \\
\hline \hline \multirow{3}{*}{ LOS } & Head & 0.90 & 0.61 & 1.12 & 1.56 & 140.5 & 2 & 1.28 & 1.56 & 108.3 & 1 \\
& Hand & 0.91 & 0.59 & 1.19 & 1.53 & 100.2 & 2 & 1.33 & 1.53 & 77.7 & 1 \\
& Pocket & 0.99 & 0.40 & 3.02 & 1.31 & 94.3 & 1 & 2.00 & 1.31 & 65.4 & 2 \\
\hline \multirow{3}{*}{ NLOS } & Head & 0.87 & 0.65 & 0.90 & 1.60 & 148.5 & 2 & 1.21 & 1.60 & 118.2 & 1 \\
& Hand & 0.93 & 0.57 & 1.35 & 1.50 & 99.6 & 2 & 1.38 & 1.50 & 75.5 & 1 \\
& Pocket & 0.85 & 0.66 & 0.83 & 1.61 & 212.6 & 2 & 1.19 & 1.61 & 171.8 & 1 \\
\hline \hline
\end{tabular}
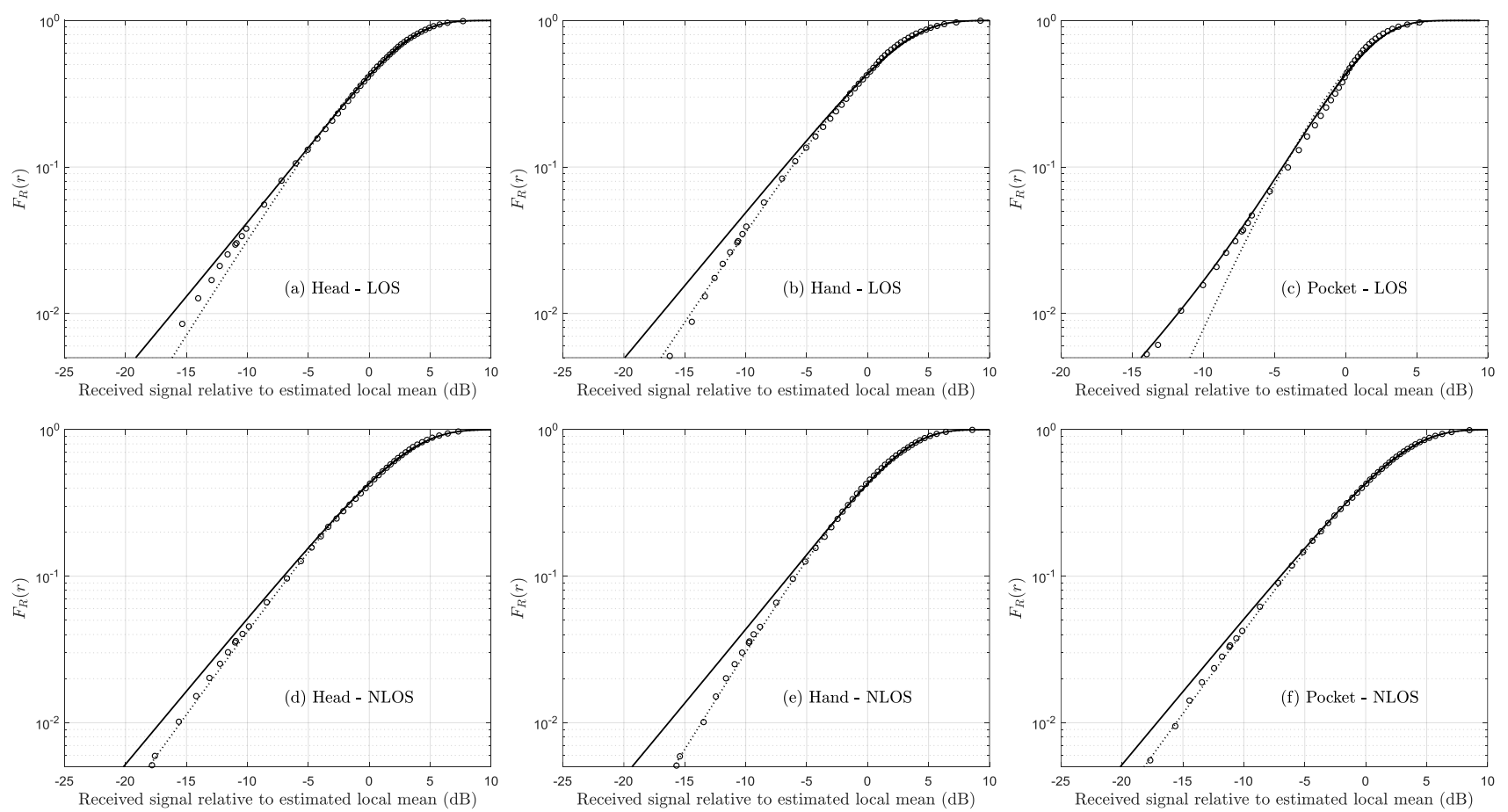

Fig. 3: Empirical CDFs (symbols) of the small-scale fading with the corresponding Rice (continuous lines) and Nakagami-m (dotted lines) CDFs for the LOS walking scenarios during the $2^{\text {nd }}$ trial and NLOS walking scenarios during the $1^{\text {st }}$ trial.

positions were shadowed or partially shadowed by the user's body and thus the Nakagami- $m$ fading model was selected in all of the NLOS cases.

All crossing rates and fade durations were calculated in steps of $0.1 \mathrm{~dB}$ in the threshold range -20 to $10 \mathrm{~dB}$. Fig. 4 shows the empirical LCRs and theoretical fitted Rice and Nakagami-m LCRs for the LOS channel conditions during the $2^{\text {nd }}$ trial whereas Fig. 5 shows the empirical AFDs and theoretical fitted Rice and Nakagami- $m$ AFDs for the NLOS channel conditions during the $1^{\text {st }}$ trial. As shown in Figs. 4 and 5, for all of the UE positions, the theoretical LCRs and AFDs provided an adequate fit for the UE-to-AP channels. As expected, the Nakagami- $m$ LCRs provided a better fit than the Rice LCRs for the head and hand positions (especially for signal levels above the $-10 \mathrm{~dB}$ threshold level) while the Rice LCR outperformed the Nakagami-m LCR for the pocket position. The maximum Doppler shifts $\left(f_{m}\right)$ for all UE positions over the LOS and NLOS conditions were es- timated by minimizing the distance between the empirical and theoretical LCR plots in a least squares sense and are presented in Table I. This parameter is normally employed to describe the time varying nature of the channel in a smallscale region. All estimated values of $f_{m}$ were found to be in the range 62.9-226.3 Hz. When comparing UE positions, the pocket and hand positions (the highest $K$ and $m$ values) had the smallest $f_{m}$ for the LOS and NLOS channel conditions, respectively.

\section{CONCLUSion}

The small-scale fading observed in short range indoor channels between a UE and a ceiling-mounted AP operating at $60 \mathrm{GHz}$ was characterized considering both first- and secondorder statistics. Over all of the measurements, the fading observed in the UE-to-AP channels was well modeled by the Rice and Nakagami- $m$ distributions. Using the $A I C_{c}$, it has 

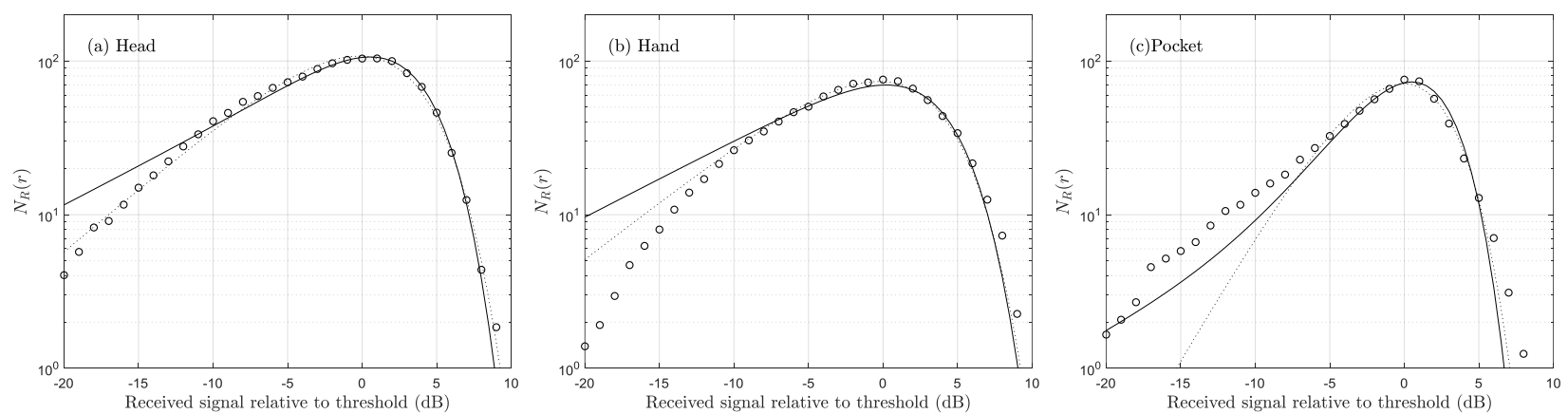

Fig. 4: Empirical (symbols) and theoretical Rice (continuous lines) and Nakagami- $m$ (dotted lines) LCRs for the LOS walking scenarios with (a) head, (b) hand and (c) pocket positions during the $2^{\text {nd }}$ trial.
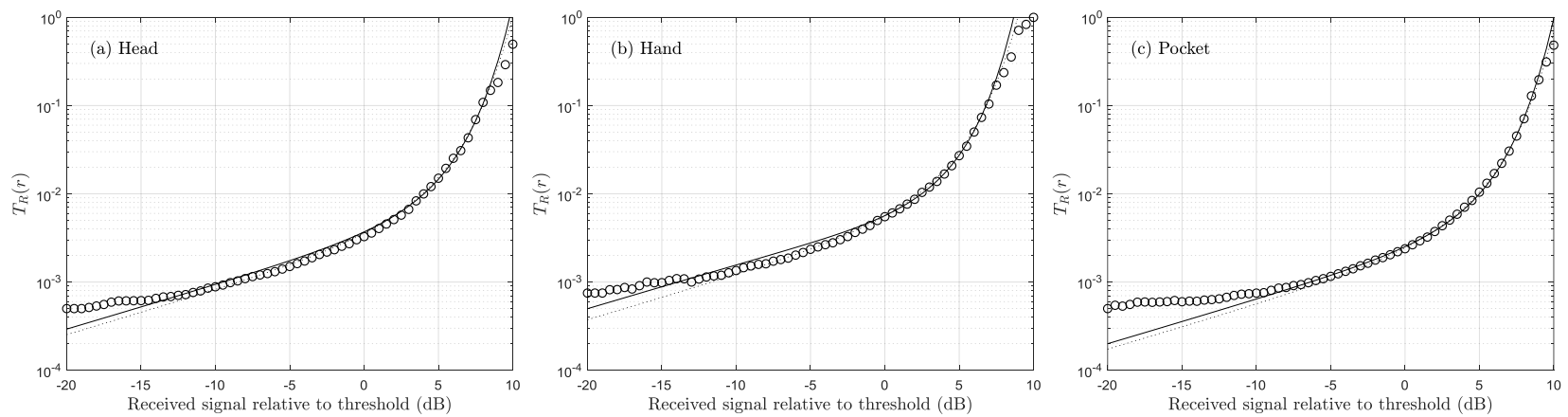

Fig. 5: Empirical (symbols) and theoretical Rice (continuous lines) and Nakagami- $m$ (dotted lines) AFDs for the NLOS walking scenarios with (a) head, (b) hand and (c) pocket positions during the $1^{\text {st }}$ trial.

been shown that the Nakagami- $m$ distribution was selected as the best model for the majority of the cases (with only one exception). The partial obscuration of the direct signal path by the user's hand may have promoted signal reception through multipath as well as any LOS component which may was present. Because of this, the Nakagami- $m$ channel was favored for two of the LOS cases. Due to this observation and the propagation geometry, we recommend that in the future more general models such as the $\kappa-\mu$ fading model [12], which can fully take into account these effects, be investigated for this type of scenario. For the NLOS cases, the user's body, combined with the hand for the hand and head cases, meant that the Nakagami- $m$ model was selected in all cases. Furthermore, it was found that these suffered slightly less severe fading than that experienced in Rayleigh fading channels. Additionally, it has been shown that the Rice and Nakagami- $m$ LCRs and AFDs provided a good fit to measurement data with all of the estimated values of $f_{m}$ were found to be in the range $62.9-226.3 \mathrm{~Hz}$.

\section{ACKNOWLEDGMENT}

This work was supported by the Engineering and Physical Sciences Research Council under Grant Reference EP/L026074/1 and the Department for Employment and Learning Northern Ireland through Grant Reference USI080.

\section{REFERENCES}

[1] S. Rangan, T. S. Rappaport, and E. Erkip, "Millimeter-wave cellular wireless networks: Potentials and challenges," Proc. IEEE, vol. 102, no. 3, pp. 366-385, Mar. 2014.

[2] C. Park and T. S. Rappaport, "Short-range wireless communications for next-generation networks: UWB, $60 \mathrm{GHz}$ millimeter-wave WPAN, and ZigBee," IEEE Wireless Commun., vol. 14, no. 4, Aug. 2007.

[3] S. K. Yoo, S. L. Cotton, Y. J. Chun, W. G. Scanlon, and G. A. Conway, "Channel characteristics of dynamic off-body communications at $60 \mathrm{GHz}$ under line-of-sight (LOS) and non-LOS conditions," IEEE Antennas Wireless Propag. Lett., vol. 16, pp. 1553-1556, Feb. 2017.

[4] S. K. Yoo, S. L. Cotton, R. W. Heath, and Y. J. Chun, "Measurements of the $60 \mathrm{GHz}$ UE to eNB channel for small cell deployments," IEEE Wireless Commun. Lett., vol. 6, no. 2, pp. 178-181, Apr. 2017.

[5] J. Reig, M.-T. Martínez-Inglés, L. Rubio, V.-M. Rodrigo-Penarrocha, and J.-M. Molina-García-Pardo, "Fading evaluation in the $60 \mathrm{GHz}$ band in line-of-sight conditions," Int. J. Antennas Propag., pp. 1-12, Aug. 2014.

[6] H. S. Wang and N. Moayeri, "Finite-state Markov channel - a useful model for radio communication channels," IEEE Trans. Veh. Technol., vol. 44, no. 1, pp. 163-171, Feb. 1995.

[7] Red Pitaya Notes, visited on 10/21/2017. [Online]. Available: http:// pavel-demin.github.io/red-pitaya-notes/.

[8] S. L. Cotton and W. G. Scanlon, "A statistical analysis of indoor multipath fading for a narrowband wireless body area network," in Proc. PIMRC, Sep. 2006, pp. 1-5.

[9] G. L. Stuber, Principles of mobile communication. Springer Science \& Business Media, 2011.

[10] M. D. Yacoub, J. V. Bautista, and L. G. de Rezende Guedes, "On higher order statistics of the Nakagami-m distribution," IEEE Trans. Veh. Technol., vol. 48, no. 3, pp. 790-794, May 1999.

[11] S. K. Yoo and S. L. Cotton, "Composite fading in non-line-of-sight off-body communications channels," in Proc. EuCAP, Mar. 2017, pp. 286-290.

[12] M. D. Yacoub, "The $\kappa-\mu$ distribution and the $\eta-\mu$ distribution," IEEE Antennas Propag. Mag., vol. 49, no. 1, pp. 68-81, Feb. 2007. 